\title{
STRATEGIES FOR BLOODLESS PRIMING OF CARDIOPULMONARY BYPASS CIRCUITS IN PAEDIATRIC CARDIAC SURGERY
}

\author{
Duc Hoang Doan*, Luong Tan Nguyen**
}

\begin{abstract}
Bloodless cardiopulmonary bypass in paediatric cardiac surgery in patients weighing more than $15 \mathrm{~kg}$ can be performed safely and effectively. Incorporating a low-prime volume oxygenator into a revised circuit reduced the need for blood transfusion and allowed us to achieve our desire flow rate of $2.6 \pm 0.21 \mathrm{~L} / \mathrm{min} / \mathrm{m}^{2}$ while maintaining a haematocrit ranging from $28 \%$ to $36 \%$.
\end{abstract}

Keywords: cardiopulmonary bypass (CPB), congenital heart disease (CHD).

\section{INTRODUCTION}

Open heart surgery with cardiopulmonary bypass without the use of blood or blood products (bloodless surgery) is now a big challenge for perfusionists, especially in the field of paediatric cardiac surgery [1] and is even more challenging in patients who follow Jehovah's Witness beliefs $[2,3]$. The aim of avoiding blood transfusion and limiting the use of blood products or coagulant factors during paediatric cardiac surgery could not be achieved if there is extreme haemodilution in extracorporeal circulation while this phenomenon is common in paediatric patients $[2,3]$. Haemodilution due to the use of a big priming volume to fill in the extracorporeal circulation compared with the considerably small circulation volume in children as well as the blood loss during surgery may dilute the blood even more.

In this study, we presented some strategies to reduce priming volume in children weighing over $15 \mathrm{~kg}$ with indications for open heart surgical repair of congenital heart anomalies such as atrial septal defects, ventricular septal defect, pulmonary artery stenosis, double outlet right ventricle, partial or total atrioventricular canal defect, tetralogy of Fallot, etc.

\section{STUDY METHODS AND RESULTS}

A total of 86 paediatric patients diagnosed with congenital heart disease whose weights were from $15-20 \mathrm{~kg}$ had indications for cardiac surgery from April 2016 to December 2018 at Vinmec Central Park Hospital to repair the defects such as atrial septal defect, ventricular septal defect, double outlet right ventricle, partial or total atrioventricular canal defect, tetralogy of Fallot, etc. These patients had a mean weight of $18.7 \pm 1.9 \mathrm{~kg}$, mean height of $109 \pm 11 \mathrm{~cm}$, and mean body skin area of $0.75 \pm 0.07 \mathrm{~m}^{2}$.

The prespecified objective was: avoiding the use of blood or blood products during and after surgery but the system was equipped with a cellsaver machine that was continuously occupied during surgery. According to our hospital's protocol, the mean bypass flow rate must reach $2.6 \pm 0.24 \mathrm{1} / \mathrm{min} / \mathrm{m}^{2}$ and the lowest estimated haematocrit (Hct) during cardiopulmonary bypass is $28 \%$. The threshold for lowest Hct $=28 \%$ during cardiopulmonary bypass is acceptable per our centre's protocol. The mean estimated blood volume of the study cohort was $1496 \pm 102 \mathrm{~mL}$. The mean preoperative haemoglobin level was $11.6 \pm 0.12 \mathrm{~g} / \mathrm{dLand}$ mean preoperative Hct was $36 \pm 2.6 \%$.

* Saigon Tam Tri Hospital

** Vinmec Central Park Hospital

Scientific director: Doan Duc Hoang MD.PhD

Manuscript received: 01/02/2019-Accepted for publication: 23/03/2020

Scientific reviewer: A/ Prof. Dang Ngoc Hung, PhD

Prof. Le Ngoc Thanh, PhD 
The conventional configuration of our cardiopulmonary bypass circuits used for children weighing over $15 \mathrm{~kg}$ requires a priming volume of $315 \mathrm{~mL}$ (Table 1) and the respective Hct level after dilution is $28 \pm 3.1 \%$ when cardiopulmonary bypass starts. As we aimed at a Hct value of $>28$ $\%$, the circuits needed to be modified. Here we present some measures taken to minimise the priming volume and the resulting parameters:
Usually, we use 3/8-inch-line for venous blood and 1/4-inch-line for arterial blood. However, we modified the system by reducing the size of the venous line to $1 / 4$-inch with the addition of a suctioning system to assist the venous blood circulation with mild pressure $(-5 \mathrm{mmHg})$ to improve venous blood return [4]. This circuits modification helped to reduce priming volume from $315 \pm 25 \mathrm{~mL}$ to $210 \pm 15 \mathrm{~mL}$ (Table 1 ).

Table 1.Differences between the conventional and modified cardiopulmonary bypass circuits

\begin{tabular}{|l|l|l|}
\hline Equipment & Conventional circuits & Modified circuits \\
\hline Oxygenator & Dideco D101(87 $\mathbf{~ L ~})$ & Medtronic Affinity Pixie $(\mathbf{4 8} \mathbf{~ m L})$ \\
\hline Arterial filter & Dideco D 131(28 $\mathbf{~ L ~})$ & None \\
\hline $\begin{array}{l}\text { Sizes of arterial }- \\
\text { venous lines }\end{array}$ & $1 / 4-\mathbf{3 / 8}$ inch & $1 / 4-\mathbf{1} / \mathbf{4}$ inch \\
\hline $\begin{array}{l}\text { Size of blood line } \\
\text { through the pump }\end{array}$ & $1 / 4$ inch & $1 / 4$ inch \\
\hline Priming volume & $\mathbf{3 1 5} \mathbf{~ m L}$ & $\mathbf{2 1 0}$ \\
\hline
\end{tabular}

- The Affinity Pixie (Medtronic Inc., - We used 12F-14FDLP aortic cannula Minneapolis, MN) oxygenator used for all 86 (Medtronic Inc., Minneapolis, MN) for arteries study subjects has features suitable for bloodless surgery such as very low static priming volume of $48 \mathrm{~mL}$ but high flow limit $(2.0 \mathrm{l} / \mathrm{min})$. We used Affinity Pixie oxygenators instead of the conventional Dideco 101 (Sorin Group, Milan, Italy) oxygenators because the latter has higher static priming volume of $87 \mathrm{~mL}$. We usedPlasmaLyte A solution (Baxter Healthcare Corporation, Deerfield, IL), a crystalloid fluid, as priming solution together with 1,100 unit of heparin and $6-10 \mathrm{mEq}$ sodium bicarbonate. A total of $2.2-$ $2.5 \mathrm{~g}$ of mannitol was used immediately after the beginning of cardiopulmonary bypass. We limited the use of albumin for priming as well as for fluid resuscitation as there are some reports on its association with acute kidney injury and postoperative bleeding [5]. and $16 \mathrm{~F}-18 \mathrm{~F}$ cannula for veins. We used the Stockert System (Stockert Cardiovascular) cardiopulmonary bypass whose pump heads could be moved easily.

- For children weighing over $15 \mathrm{~kg}$, we usually use big pump head that requires a conductor line with the respective length. However, to reduce the priming volume, we experimented smaller pump heads to shorten the line. With this method, the connecting line from the reservoir to the oxygenator was reduced to $26 \pm 1$.4inches compared with the conventional length of $33 \pm 2.2$ inches.

- We used small pump heads to suction blood from the surgical field or heart chambers. These heads were appropriately located so that the distance to the surgical table was shortest and the 
length of the line to the oxygenator was minimised. We used the small size lines (1/4inch) for all blood suctioning pumps except for the line used to suction blood from the aorta which has a diameter smaller than usual (1/8inch) to prevent blood loss from the extracorporeal circulation because this line must be maintained filled during de-airing phase to prepare for heart re-beating after all surgical repairs are completed. We also considered using the 3/16-inch lines for suctioning blood but there is a risk of hemolysis, especially in patients with many collateral circulations that require higher than normal suctioning speed.

- With oxygenators with suitable design such as Affinity Pixie, and especially those with integrated arterial filter membrane inside, removing the external filter from the bypass circuits could further reduce priming volume by $28 \mathrm{~mL}$ (the priming volume required to fill the external arterial filter). Doctors need to make sure that parents and other family members understand the surgical protocol and strategies for bloodless cardiopulmonary bypass. However, our hospital always has a back-up protocol that allows rapid indication and transfusion of blood in emergency circumstances [2].

- During surgery, the technician set up the cardiopulmonary bypass through the arterialvenous connection must cut the connection line to reduce its length and therefore, reducing a priming volume by $33 \pm 2.3 \mathrm{~mL}$. After connecting all venous cannula to the blood lines, we also reducea volume $23 \pm 2,0 \mathrm{~mL}$ of the priming solution from the circuitright before starting cardiopulmonary bypass, after the surgeon opens the venous clamp. So, the shortening of the arterial-venous connection and the reduction of priming solutionright before cardiopulmonary bypass reduced the priming volume (already reduced from $315 \mathrm{~mL}$ to $210 \mathrm{~mL}$ ) and through this measure, we further reduced the priming volume from $210 \pm 15 \mathrm{~mL}$ to $160 \pm 15 \mathrm{~mL}$.

- Regarding the study results, the study cohort had a mean preoperative Hct of $36 \pm 2,6 \%$. The mean cardiopulmonary bypass time of this group of patients was $55 \pm 9$ minutes. The lowest Hct level was $24 \pm 1,2 \%$, occurring immediately after the application of Custodial Bretschneider HTK solution (Essentials Pharm, Newtown, PA)for cardioplegia. The highest Hct level was $30 \pm 1,9 \%$. We used the HPH 400 blood filter (Minntech Mini, Minneapolis, MN) to filter the excess fluid and this filter is already integartedin the system but does not require priming until the cardiopulmonary bypass starts. The analysis of blood gases after stopping the use of protamine revealed a Hct of $32 \pm 3.4 \%$. Furthermore, right after terminating cardiopulmonary bypass, we collected the volume of blood in venous line by replacing it with a respective volume of Plasma Lyte A solution (Baxter Healthcare Corporation).This volume of blood was transfused back to the patient to further increase Hct.

- All patients were transferred to post-operative intensive care unit with a mean Hct of $33 \pm 3.1 \%$. They were treated until hemodynamically stable and weaned from ventilator. The mean postoperative ventilation time was $4 \pm 0.8$ hours. No intra- or post-operative haematuria was recorded. Arterial blood gases results were within normal limits with no indications of metabolic acidosis. Mean postoperative lactate level was $1.4 \pm 0.2 \mathrm{mmol} / \mathrm{L}$, and mean serum creatine level was $47 \pm 5.1 \mu \mathrm{mol} / \mathrm{L}$. The mean volume of blood loss through chest drainage was $75 \pm 9.5 \mathrm{~mL}$. No patient required blood or blood products transfusion postoperatively and all patients were discharged with a mean length of stay of $4 \pm 1$ days with a mean Hct of $32 \pm 2.0 \%$ on discharge. 


\section{DISCUSSIONS}

In order to mitigate the haemodilution effect of cardiopulmonary bypass, we are confident that bloodless cardiopulmonary bypass is safe for paediatric cardiac surgery in children weighing $15-20 \mathrm{~kg}$, and these strategies are suitable for patients that do not need any types of blood products. In our hospital, the priming volume of cardiopulmonary bypass circuit in patients whose weights are from $2-14 \mathrm{~kg}$ is $170-205 \mathrm{~mL}$. In patients weighing over $15 \mathrm{~kg}$, a bigger system is used and therefore an additional $105 \mathrm{~mL}$ of priming volume is necessary.

With low-priming volume oxygenators, we could move the pump heads of the bypass machine closer to the surgical field, and by using smaller venous line in combination with the use of lowpressure suctioning system to assist venous blood return, we could reduce priming volume, and all of these techniques help to reduce the haemodilution effects. The cut of connecting lines also help to maintain more blood return to reservoir and therefore ensuring the blood volume going through the haemoconcentrator system so that after terminating cardiopulmonary bypass, we could achieve higher Hct. The combination of the above-mentioned methods helps to limit the use of blood or blood products and accordingly save considerable resources and most importantly, achieve the goals for paediatric patients of having postoperative Hct level high enough to reduce the need for blood transfusion after surgery.

In addition, the use of $1 / 8$-inch line to suction the blood in heart chambers is the main factor to maintain circulation volume. The continuous micro-haemofilter technique is helpful to remove excessive fluid during cardiopulmonary bypass. To design a modified microfilter into the systemic circulation is a significant challenge due to the need of additional priming volume while our target is to limit volume at the termination phase of cardiopulmonary bypass. Our overall aim is to perform bloodless paediatric cardiac surgery as much as possible. After this study, we will implement similar strategies but also design a new arterial filter into the circuits for children weighing over $15 \mathrm{~kg}$ and at the moment, $60 \%$ of open-heart surgeries in our hospital are bloodless, even in patients with high risk of bleeding such as re-operation.

\section{CONCLUSIONS}

Cardiac surgery in paediatric patients weighing over $15 \mathrm{mg}$ with bloodless cardiopulmonary bypass can be performed safely. The combination of low-priming volume oxygenator with modified cardiopulmonary bypass circuits helps to reduce the need of blood transfusion and allows us to achieve optimal circulation volume with a mean flow rate of $2.6 \pm 0.21 \mathrm{1} / \mathrm{min} / \mathrm{m}^{2}$ while still maintaining the variation of Hct values in the normal range of $28 \%-36 \%$.All patients in our study were discharged early and the average length of stay was $4 \pm 1$ days and the mean Hct level was $32 \pm 2.0 \%$.

\section{REFERENCES}

5. Miyaji KA, Kohira SA, Miyamoto TA, et al. Paediatric cardiac surgery without homologous blood transfusion, using a miniaturized bypass system in infants with lower body weight. J Thorac Surg. 2007;134:284-9.

6. Forest RJ, Groom RC, Quinn R, Donnelly J, Clark C. Repair of hypoplastic left heart syndrome in a $4.25 \mathrm{~kg}$ Jehovah's Witness. Perfusion. 2002;17:221-5.

7. Karimi MO, Florentino-Pineda IV, Weatherred TE, et al. Blood conservation operations in paediatric cardiac patients: A paradigm shift of blood use. Ann Thorac Surg. 2013;95:962-7.

8. Nakanishi K, Shichijo T, Shinkawa Y, et al. Usefulness of vacuum-assisted cardiopulmonary bypass circuit for paediatric open-heart surgery in reducing homologous blood transfusion. Eur $\mathrm{J}$ Cardiothorac Surg. 2001; 20:233-8.

9. Utley JR, Stephens DB, Wachtel C, et al. Effect of albumin and mannitol on organ blood flow, oxygen delivery, water content and renal function during hypothermic haemodilution cardiopulmonary bypass. Ann Thorac Surg. 1982;33:250-7. 